\begin{tabular}{|c|c|}
\hline Title: & $\begin{array}{l}\text { Online Parameter Identification of Permanent Magnet Synchronous Machines with } \\
\text { Nonlinear Magnetics based on the Inverter Induced Current Slopes and the dq-System } \\
\text { Equations }\end{array}$ \\
\hline Authors: & $\begin{array}{l}\text { Simon Decker, Johannes Stoss, Andreas Liske, Matthias Brodatzki, Johannes Kolb, } \\
\text { Michael Braun }\end{array}$ \\
\hline Institute: & $\begin{array}{l}\text { Karlsruhe Institute of Technology (KIT) } \\
\text { Institute of Electrical Engineering (ETI) }\end{array}$ \\
\hline Type: & Conference Proceedings \\
\hline Published at: & $\begin{array}{l}\text { Proceedings } 2019 \text { European Conference on Power Electronics and Applications } \\
\text { (EPE'19 ECCE Europe), Genova, Italy, } 2019 \\
\text { Publisher: IEEE } \\
\text { Year: } 2019 \\
\text { ISBN: } 978-9-0758-1530-6\end{array}$ \\
\hline Hyperlinks: & DOI: $\underline{10.23919 / E P E .2019 .8915019}$ \\
\hline
\end{tabular}

(c) 2019 IEEE. Personal use of this material is permitted. Permission from IEEE must be obtained for all other uses, in any current or future media, including reprinting/republishing this material for advertising or promotional purposes, creating new collective works, for resale or redistribution to servers or lists, or reuse of any copyrighted component of this work in other works. 


\title{
Online Parameter Identification of Permanent Magnet Synchronous Machines with Nonlinear Magnetics based on the Inverter Induced Current Slopes and the dq-System Equations
}

\author{
Simon Decker (iD), Johannes Stoss, Andreas Liske, Matthias Brodatzki, Johannes Kolb*, \\ Michael Braun \\ Karlsruhe Institute of Technology (KIT) \\ Elektrotechnisches Institut (ETI) - Electrical Drives and Power Electronics \\ Kaiserstr. 12, 76131 Karlsruhe, Germany \\ E-Mail: simon.decker@kit.edu \\ URL: http://www.eti.kit.edu \\ *Schaeffler Technologies AG \& Co. KG - SHARE at KIT
}

\section{Keywords}

«Synchronous motor», «Estimation technique», «Adjustable speed drive», «Adaptive control»

\begin{abstract}
This paper shows a fast online parameter identification method for permanent magnet synchronous machines (PSMs), which uses the PWM (pulse width modulation) excitations and no additional test signals. Based on the inverter induced current slopes, the corresponding applied voltages, the rotor angle and a precise machine model, the model's parameters are calculated. Thereby the machine model consists of the dq-system equations, linearized within one PWM period. For stable and precise identification of the PSM machine parameters, the machine model has to be modified with a regularization approach. The identified parameters enable self-commissioning, tuning of the control parameters, condition monitoring or inner fault detection. In this paper the theoretical approach of the suggested method and simulation results of an equivalent test-bench system are presented.
\end{abstract}

\section{Introduction}

Electrical machines nowadays are designed with low weight, small size and high power. These machines are commonly permanent magnet synchronous machines (PSMs). The high utilization of these machines yields nonlinear magnetics due to saturation and cross-coupling effects. Stable, accurate and dynamic control of high performance drives is only possible, if these effects are considered in the control. Furthermore, condition monitoring, online tuning of the control parameters, self-commissioning and fault detection are topics of increasing importance. Evaluation of these topics also demands the knowledge of precise machine parameters, including saturation and cross coupling, during operation and startup. Therefore, the online parameter identification is under research. In [1] is shown that, even for linear PSMs, it is not possible to identify all parameters at steady state and dynamic operation online without previous knowledge of the machine, test signals and/or special, extended algorithms. As a result, various identification approaches, often with test signal use, based on control theory and system theory were investigated in the past.

Pure observer based methods without test signals are e.g. Kalman Filters [1] or Model Reference Adaptive Systems (MRAS). More modern algorithms derived from computer science are also examined. Typical for these algorithms are Particle Swarm Algorithms or Genetic Algorithms [2]. These methods are often based on a linear set of parameters, computationally extensive and have inferior dynamics compared to other approaches. Results can be obtained faster with test signal use. Typical are low frequency voltage or current signal injection during operation or at startup. But these voltage or current signal injections lead to torque ripple, additional losses and noise at operation. Therefore, these methods are mostly used for self-commissioning systems or for short time adjusting of e.g. the control parameters. 
An overview over different self-commissioning algorithms is given in [3]. Principles with less additional disturbance and a fast response are based on high frequency current or voltage signal injection. However, for these identification schemes, extensive signal processing systems with consideration of the high frequency effects of the electrical machine and the inverter are necessary. Special and complex filtering algorithms, etc. are required [4]. Even with these high frequency signal injection additional losses, disturbances, etc. are present.

Methods without additional disturbances due to test signals, besides the pure observer based methods as described before, use the evaluation of the existing two level inverter induced current slopes. These always existing "test signals" are often named "pulse width modulation (PWM) excitation". In encoderless control algorithms the evaluation of the PWM excitation is often used for calculation of the rotor angle. Both, the calculation of the rotor angle and the machine parameter identification have similar requirements. The challenging and extensive part of these methods is the fast and precise measurement of the currents, especially the current derivatives [5] for the fast and robust calculation without test signals. Different evaluation methods of the current derivatives are possible [6]. In [7], the PSM is field oriented controlled with continuous space vector modulation and the measured and highly oversampled absolute current values are fitted with a least-squares algorithm to get the current derivatives from the PWM excitation. Based on these fitted current derivatives the position estimation, parameter and control integration with a simplified three phase machine model without resistance is shown. Due to the minimum pulse width at high inverter switching frequency the dynamic of these introduced algorithm is limited due to the AD-converter dynamics, even with fast AD-converters. Another fast calculation approach, similar to the one presented in [7], is theoretically analyzed and discussed in [8]. Thereby the current derivatives, induced by the discontinuous Direct Torque Control scheme are directly evaluated to calculate the linear supposed dq-inductances.

Compared to the ideas before, this paper shows a method to calculate the nonlinear PSM model, as derived in [9] for offline measurements, direct and independently in each point of operation. In the following the theoretical method for calculating the nonlinear parameters including the stator resistance of the PSM from the currents, the current derivatives and the applied voltages without additional testsignal, is shown. Therefore, the dq-system equations are linearized in a small-signal behavior within a fast control period $T_{\mathrm{C}}$. Considering the switching-states of the inverter, the actual point of operation and the measured values within a control period $T_{\mathrm{C}}$ a system of linear equations is created. For robust parameter identification it is necessary that the inverse problem of these linear equations is a solvable system. Therefore, the solvability of the system equations is investigated based on a quality criterion, the condition number of the matrix. Badly conditioned problems during identification are enhanced with mathematical regularization of the parameter calculation problem in the simulation, also further methods to increase the solvability are discussed.

At first, the rotor oriented nonlinear PSM model is derived. After that, the basic idea of the identification algorithm is motivated and the necessary formulas and boundary conditions are given. A possible target system for further test-bench implementations is afterwards defined and parametrized in an acausal Matlab/Simulink/Simscape simulation environment. Based on this simulation environment the simulations of the identification at a certain operating point are shown to validate the derived algorithm.

\section{PSM Model}

\section{Time continuous model}

Permanent magnet synchronous machines, especially for control and simulation, are often described in the rotor oriented dq-frame. Thereby, three symmetric, star-connected stator windings are assumed. The neutral-point of the machine is not connected. As a result, current-, flux- and voltage-zero-components disappear. Friction losses, skin and proximity effects as well as iron losses are neglected because of the small impact on machine control. Furthermore, dielectric currents, thermal dependencies and ageing effects, are not considered in the model. Considering of e.g. ageing effects of the permanent magnets is possible by monitoring the long term behavior of the introduced system equations. The flux-linkages are averaged over the rotor angle; therefore, the spatial harmonics are not included. The stator voltage equations are derived from Ohm's law, Kirchhoff's law and the equations of Maxwell. The transformation from the stator-oriented three phase system to the rotor-oriented dq-reference frame yields the PSM system equations: 


$$
\begin{aligned}
& v_{\mathrm{d}}=R i_{\mathrm{d}}+\frac{\mathrm{d} \psi_{\mathrm{d}}\left(i_{\mathrm{d}}, i_{\mathrm{q}}\right)}{\mathrm{d} t}-\omega \psi_{\mathrm{q}}\left(i_{\mathrm{d}}, i_{\mathrm{q}}\right) \\
& v_{\mathrm{q}}=R i_{\mathrm{q}}+\frac{\mathrm{d} \psi_{\mathrm{q}}\left(i_{\mathrm{d}}, i_{\mathrm{q}}\right)}{\mathrm{d} t}+\omega \psi_{\mathrm{d}}\left(i_{\mathrm{d}}, i_{\mathrm{q}}\right)
\end{aligned}
$$

The ohmic resistance of the stator windings is $R$, the electric frequency is $\omega$. The voltage- and currentcomponents of the direct- and quadrature-axis are $v_{x}, i_{x}$ with $x \in\{\mathrm{d}, \mathrm{q}\}$. The current dependent stator flux-linkages $\psi_{x}$ contain the anisotropy of the rotor, the saturation of the iron as well as dynamic crosscoupling effects between the direct- and quadrature-axis of the machine.

The partial derivation of $\psi_{x}$ leads to the differential inductances $L_{x x}$. The inductances $L_{\mathrm{dd}}, L_{\mathrm{qq}}$ denote the self-inductances in direct- and quadrature-axis, $L_{\mathrm{dq}}, L_{\mathrm{qd}}$ are the mutual inductances [9]. The reciprocity relations for the mutual inductances yields $L_{\mathrm{dq}}=L_{\mathrm{qd}}$ [10]. In the following only $L_{\mathrm{dq}}$ is considered. In (3) and (4) the dynamic component of the flux-linkages $\mathrm{d} \psi_{\mathrm{d}} / \mathrm{d} t$ and $\mathrm{d} \psi_{\mathrm{q}} / \mathrm{d} t$ are expressed with the derived current dependent inductances.

$$
\begin{aligned}
& v_{\mathrm{d}}=R i_{\mathrm{d}}+L_{\mathrm{dd}}\left(i_{\mathrm{d}}, i_{\mathrm{q}}\right) \frac{\mathrm{d} i_{\mathrm{d}}}{\mathrm{d} t}+L_{\mathrm{dq}}\left(i_{\mathrm{d}}, i_{\mathrm{q}}\right) \frac{\mathrm{d} i_{\mathrm{q}}}{\mathrm{d} t}-\omega \psi_{\mathrm{q}}\left(i_{\mathrm{d}}, i_{\mathrm{q}}\right) \\
& v_{\mathrm{q}}=R i_{\mathrm{q}}+L_{\mathrm{qq}}\left(i_{\mathrm{d}}, i_{\mathrm{q}}\right) \frac{\mathrm{d} i_{\mathrm{q}}}{\mathrm{d} t}+L_{\mathrm{qd}}\left(i_{\mathrm{d}}, i_{\mathrm{q}}\right) \frac{\mathrm{d} i_{\mathrm{d}}}{\mathrm{d} t}+\omega \psi_{\mathrm{d}}\left(i_{\mathrm{d}}, i_{\mathrm{q}}\right)
\end{aligned}
$$

\section{Time discrete model}

The implementation of control algorithms on digital signal processing systems is time-discrete. When the inertia of the machine is sufficiently large and/or the control period $T_{\mathrm{C}}$ is short compared to the machine's time constants, the electric frequency $\omega$ can be assumed constant during one control period. With evaluation of the electrical parameters within a short control period a small signal model, e.g. derived in [11] or [12], can be assumed. The self- and mutual-coupling inductances and the ohmic stator resistance are presumed to be constant within this control period $T_{\mathrm{C}}$. The flux-linkages in (3) and (4) are considered piecewise linear:

$$
\psi_{x}\left(i_{\mathrm{d}_{t_{n+1}}}, i_{\mathrm{q}_{t_{n+1}}}\right)=\psi_{x}\left(i_{\mathrm{d}_{t_{n}}}, i_{\mathrm{q}_{t_{n}}}\right)+L_{x x}\left(i_{\mathrm{d}_{t_{n, \ldots n+3}}}, i_{\mathrm{q}_{t_{n, \ldots n+3}}}\right) \cdot \frac{i_{x_{t_{n+1}}}-i_{x_{t_{n}}}}{2} \text { with } x \in\{\mathrm{d}, \mathrm{q}\}
$$

The trapezoidal rule, shown in [13], describes the time-discrete voltage distribution. Together with (3) and (4) the piecewise linear stator voltages can be expressed with the equations (6) and (7).

$$
\begin{aligned}
& v_{\mathrm{d}_{t_{n}, t_{n+1}}}=\frac{R}{2}\left(i_{\mathrm{d}_{t_{n}}}+i_{\mathrm{d}_{t_{n+1}}}\right)+L_{\mathrm{dd}}\left(i_{\mathrm{d}_{t_{n}, t_{n+1}}} i_{\mathrm{q}_{t_{n}, t_{n+1}}}\right) \cdot \frac{i_{\mathrm{d}_{t_{n+1}}}-i_{\mathrm{d}_{t_{n}}}}{t_{n+1}-t_{n}}+L_{\mathrm{dq}}\left(i_{\mathrm{d}_{t_{n} t_{n+1}}}, i_{\mathrm{q}_{t_{n} t_{n+1}}}\right) \cdot \frac{i_{\mathrm{q}_{t_{n}+1}}-i_{\mathrm{q}_{t_{n}}}}{t_{n+1}-t_{n}} \\
& -\omega_{t_{n}, t_{n+1}}\left(\psi_{\mathrm{q}}\left(i_{\mathrm{d}_{t_{n}}}, i_{\mathrm{q}_{t_{n}}}\right)+L_{\mathrm{dq}}\left(i_{\mathrm{d}_{t_{n} t_{n+1}}}, i_{\mathrm{q}_{t_{n} t_{n+1}}}\right) \cdot \frac{i_{\mathrm{d}_{n+1}}-i_{\mathrm{d}_{t_{n}}}}{2}+L_{\mathrm{qq}}\left(i_{\mathrm{d}_{t_{n} t_{n+1}}}, i_{\mathrm{q}_{t_{n}, t_{n+1}}}\right) \cdot \frac{i_{\mathrm{q}_{t_{n+1}+1}}-i_{\mathrm{q}_{t_{n}}}}{2}\right) \\
& v_{\mathrm{q}_{t_{n}, t_{n+1}}}=\frac{R}{2}\left(i_{\mathrm{q}_{t_{n}}}+i_{\mathrm{q}_{t_{n+1}}}\right)+L_{\mathrm{qq}}\left(i_{\mathrm{d}_{t_{n}, t_{n+1}}}, i_{\mathrm{q}_{t_{n} t_{n+1}}}\right) \cdot \frac{i_{\mathrm{q}_{t_{n+1}}}-i_{\mathrm{q}_{t_{n}}}}{t_{n+1}-t_{n}}+L_{\mathrm{dq}}\left(i_{\mathrm{d}_{t_{n} t_{n+1}}}, i_{\mathrm{q}_{t_{n}, t_{n+1}}}\right) \cdot \frac{i_{\mathrm{d}_{n+1}}-i_{\mathrm{d}_{t_{n}}}}{t_{n+1}-t_{n}} \\
& +\omega_{t_{n}, t_{n+1}}\left(\psi_{\mathrm{d}}\left(i_{\mathrm{d}_{t_{n}}}, i_{\mathrm{a}_{t_{n}}}\right)+L_{\mathrm{dq}}\left(i_{\mathrm{d}_{t_{n} t_{n+1}}}, i_{a_{t_{n} t_{n+1}}}\right) \cdot \frac{i_{\mathrm{q}_{t_{n}+1}}-i_{\mathrm{q}_{t_{n}}}}{2}+L_{\mathrm{dd}}\left(i_{\mathrm{d}_{t_{n} t_{n+1}}}, i_{\mathrm{q}_{t_{n} t_{n+1}}}\right) \cdot \frac{i_{\mathrm{d}_{t_{n+1}}}-i_{\mathrm{d}_{t_{n}}}}{2}\right)
\end{aligned}
$$

Equation (6) and (7) describe now the precise discrete dq-model. The inputs are the currents $i_{x_{t_{n}}}$ and their derivatives $\frac{i_{x_{t_{n+1}}}-i_{x_{n}}}{t_{n+1}-t_{n}}$, the rotor angle $\gamma$, the electric frequency $\omega_{t_{n}, t_{n+1}}$ and the estimated voltage references $v_{x_{t_{n}, t_{n+1}}}$ from a voltage source inverter. This representation of the dq-equations is often used in discrete control algorithms and simulations.

\section{(dq-) Model for the online parameter identification}

The calculation of the online parameters with equation (6) and (7) is feasible but for the online identification, especially with unknown variables like dead-time of the semiconductors and other effects, a more robust representation is necessary. Sampling the values in the middle of each switching-state allows the simplification of the time-discrete model. Due to the small signal linearization with constant inductances and linearized flux-linkages those "middle-sampled values" of the direct measured current 
slopes are always admissible. The voltages $v_{x, t_{n}}$ with $x \in\{\mathrm{d}, \mathrm{q}\}$ are also middle-sampled values. Only the current absolute values are time-sensitive. Compared to the voltage-time failures in the discrete representation these effects are smaller. The equations for the first interval are

$$
\begin{aligned}
& v_{\mathrm{d}, t_{n}}=R \overline{i_{\mathrm{d}, t_{n}}}+L_{\mathrm{dd}} \frac{\overline{\mathrm{d} i_{\mathrm{d}, t_{n}}}}{\mathrm{~d} t}+L_{\mathrm{dq}} \frac{\overline{\mathrm{d} i_{\mathrm{q}, t_{n}}}}{\mathrm{~d} t}-\omega \overline{\psi_{\mathrm{q}, t_{n}}} \\
& v_{\mathrm{q}, t_{n}}=R \overline{\bar{i}_{\mathrm{q}, t_{n}}}+L_{\mathrm{qq}} \frac{\overline{\mathrm{d} i_{\mathrm{q}, t_{n}}}}{\mathrm{~d} t}+L_{\mathrm{qd}} \frac{\overline{\mathrm{d}} \frac{i_{\mathrm{d}, t_{n}}}{\mathrm{~d} t}+\omega \overline{\psi_{\mathrm{d}, t_{n}}}}{}
\end{aligned}
$$

The following equations of each switching state $t_{[n, \ldots, n+k]}$ for a following matrix representation can be established similarly. Thereby the flux linkages have to be considered piecewise linear as described before. Equation (10) shows this exemplarily for the d-axis flux-linkages for the time $t_{n+1}$.

$$
\psi_{\mathrm{d}, t_{n+1}} \approx \psi_{\mathrm{d}, t_{n}}+L_{\mathrm{dd}} \frac{\overline{\mathrm{d} i_{\mathrm{d}, t_{[n, n+1]}}}}{\mathrm{d} t}+L_{\mathrm{dq}} \frac{\overline{\mathrm{d} i_{\mathrm{q}, t_{[n, n+1]}}}}{\mathrm{d} t}
$$

\section{Online Parameter Identification}

The voltage equations (8) and (9) are an under-determined system of equations with two equations and six unknowns: $R, L_{\mathrm{dd}}, L_{\mathrm{dq}}, L_{\mathrm{qq}}, \psi_{\mathrm{d}}, \psi_{\mathrm{q}}$. These equations are not directly solvable. As a solution, in [14] the equations are simplified and linearized within two control periods. The parameters are calculated with consideration of the different operation points and an additive test-signal.

Another possibility is the direct use of the inverter induced current slopes from pulse width modulation instead of a test-signal. In case of space vector modulation this yields to up to seven evaluable current slopes within a control period. Thereby, six different "active switching states" and two different "freewheeling switching states" are possible. The switching states are depending on the different operation points and control strategy of the inverter. The dq-system equations of these switching states give fourteen equations within one control period. Fig. 1 shows one of these control periods. The complementary switching states, the linearized direct-axis currents and the applied voltages of the directaxis are displayed. At first the PWM excitation of this control period is considered. The current slopes show a symmetry to the center of one control period. Evaluation of all sequences or switching states yield to linear depending equations and lack of solution. Therefore, each switching state symmetric in the two dq-system equations is only used once. In space vector modulation, there are two different "active states" and one "freewheeling state" during each control period. By evaluation of two following "active states" and one "freewheeling state" the start or end of the calculation has to be a freewheeling state. If an applied voltage vector covers one of the six space vectors of space vector modulation, normally only one active state is applied. Similar behavior depending on the actual rotor position, appears if the dq-frame covers directly one of the six vectors. For control algorithms the parameters have to be identified reliable. To ensure identification also during these cases, other space vectors for the modulation or modulation schemes have to be chosen. At small duty cycles or missing evaluable switching states, the pulse pattern of the space vector modulation can be shifted to have longer evaluable "active states" and "freewheeling states" [15]. Other possibilities are the extrapolation of the small dutycycle values. This paper focuses on the main principle for the identification, in the evaluation these cases are not evaluated, which is sufficient for e.g. condition monitoring of PSMs. Assuming three consecutive, valid time steps $t_{n} \ldots t_{(n+3)}$ with two active and one freewheeling switching state yields to the six linear independent equations. With equations (8) and (9) at these defined switching states the square matrix in (11) is defined.

$$
A x=b \text { with } A \in \mathbb{R}^{m, m}, x \in \mathbb{R}^{m}, b \in \mathbb{R}^{m}
$$

The matrix $A \in \mathbb{R}^{m, m}$ at each time $t_{n}$ consists of measured direct and quadrature axis currents, their derivatives and the electric frequency. The vector $b \in \mathbb{R}^{m}$ consists of the applied direct and quadrature voltages. 


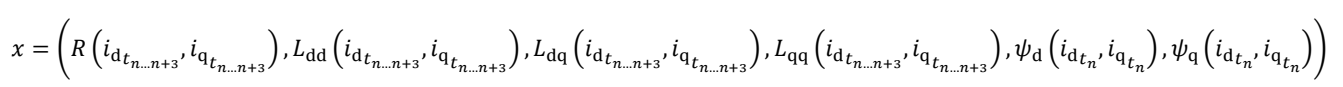

The vector $x \in \mathbb{R}^{m}$ contains the searched parameters, shown in vector (12). The inductances and the ohmic stator resistance is supposed to be constant within the considered time steps. The flux linkages are linearized within these time steps and are extrapolated to a certain current value within an evaluated control period.
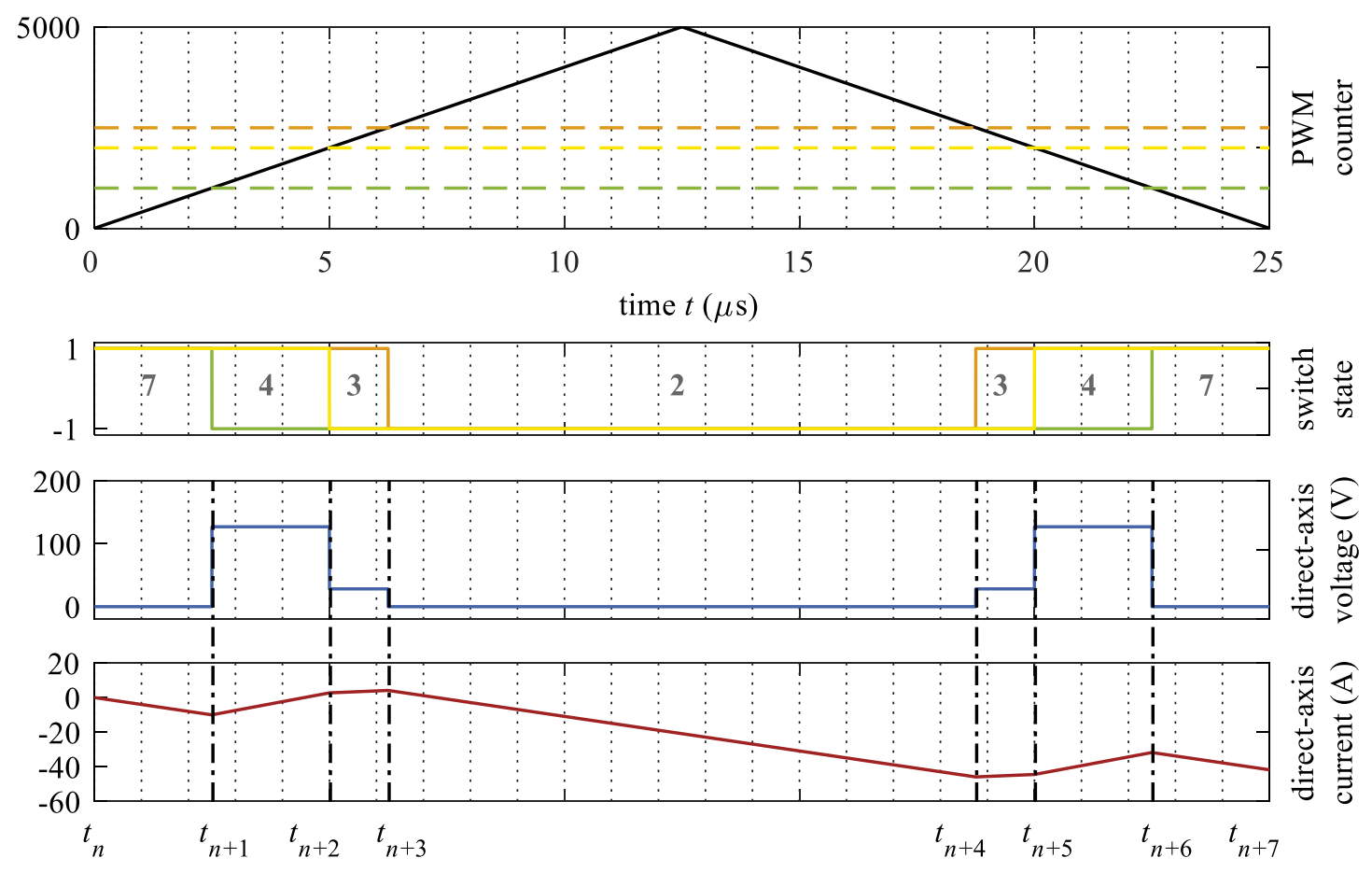

Fig. 1: One control period of the voltage stage inverter, the idealized pulses and the direct-axis currents with the PWM excitation as well as the effective direct-axis voltage. The sampling times of the currents, the current slopes and the voltages are in the middle between each pulse $\left(t_{n}\right)$. Thereby switching-states 7 and 0 indicate a "freewheeling state", 3 and 4 indicates an "active state".

The matrix of the dq-system equation is displayed in (13), it contains the dq-system equations during one control period.

$$
\underbrace{\left[\begin{array}{ccc} 
& \cdots & \\
\vdots & \ddots & \vdots \\
& \cdots &
\end{array}\right]}_{A} \cdot \underbrace{\left[\begin{array}{c}
u_{\mathrm{d}_{t_{n}}} \\
\vdots \\
u_{\mathrm{d}_{t_{[n, n+\cdots]}}} \\
u_{\mathrm{q}_{t_{n}}} \\
\vdots \\
u_{\mathrm{q}_{[n, n+\cdots]}}
\end{array}\right]}_{\left.\begin{array}{c}
R \\
L_{\mathrm{dd}} \\
L_{\mathrm{dq}} \\
L_{\mathrm{qq}} \\
\psi_{\mathrm{d}} \\
\psi_{\mathrm{q}}
\end{array}\right]}
$$

A trivial solution of the problem $A x=b$ is available, if the kernel (ker) of the matrix contains only the null vector as an element, i.e. $\operatorname{ker}(A)=\{0\}$. This means that the number zero is not an admissible eigenvalue of $A$. Solving $A x=b$ for the vector $x$ gives an inverse problem $A^{-1} A x=A^{-1} b$. The inverse $A^{-1}$ can be calculated with several algorithms like the LU-, QR-, Cholesky-, or the singular value decomposition.

Instabilities in numerical algorithms (e.g. the LU-decomposition) are caused by small or colliding eigenvalues and should be avoided. The result of the matrix decomposition yields to wrong solutions, if these instabilities are not considered. Therefore, the solvability, respectively the invertibility of these problems have to be verified. In [14] the solvability of $A$ is checked by the determinant of the matrix. 
Another criterion of small or colliding eigenvalues is the condition number which measures how sensitive a function/matrix is against their inputs or input errors. Low condition numbers $\kappa(A)$ indicate well-conditioned problems, high condition numbers $\kappa(A) \gg 1$ indicate ill-conditioned problems [16]. As a rule of thumb, if the number $\kappa(A)=10^{k}$, then you may lose roughly $k$ digits of accuracy, additional to the numerical decomposition method. With the maximal and minimal eigenvalues, $\lambda_{\max }(A)$ and $\lambda_{\min }(A)$ of a square matrix the condition number (14) can be calculated.

$$
\kappa(A)=\frac{\left|\lambda_{\max }(A)\right|}{\left|\lambda_{\min }(A)\right|}
$$

In a simulation the PSM machine is PI controlled with space vector modulation and evaluated at quasistationary operation. Reviewing the defined valid PWM switching states with enough dq-equations and without the prohibited voltage vectors at certain points of operation the matrix $A$ shows various and high condition numbers $\kappa(A) \gg 1$. This indicates still ill conditioned inverse problems. As a result, the parameters cannot be calculated reliably in the whole range due to numerical inaccuracies, even with different algorithms for solving the system of linear equations. The inverse problem itself has to be changed to obtain a more reliable solution. Therefore, a closer look onto the condition numbers is necessary. Based on the simulation results the inverse problem can be categorized as follows.

At high speed operation the eigenvalues of the flux-linkages and the inductances are close together, the eigenvalue of the resistance is responsible for the inclination of the eigenvalues and yields a bad condition number. In lower speed region, the eigenvalues are located closer together, but this inverse problem is still an ill-conditioned problem. Higher currents improve the condition number and especially the identification of the resistance. The voltage-drop over the resistance is only current depending, according to the dq-system equations (8) and (9).

Methods to improve ill conditioned problems include preconditioning, scaling or regularization. Most commonly, regularization methods are used, which are also considered in this paper. Regularization methods introduce additional information into the problem to improve the trade-off between the size of the regularized solution and the quality of the fit to the original problem.

Depending on the original problem, a regularization not always yields a better solution [17]. Neglecting the resistance identification at high speed operation yields a better condition problem. This is feasible because the contribution of the voltage of the resistance within the matrix is small. Similar behavior is appearing at low speed and low currents, the flux-linkages directly depend on the electric frequency, small values are quite sensitive for numerical issues or noise. Therefore the problem $A x=b$ can be reduced, with less equations, or the additional equations used for better solution with QR or singular value decomposition. In the other cases, a regularization approach yields reliable results. The Tikhonov regularization, e.g. with the graphical indication, the L-curve by P.C. Hansen [18], is one of them. Approximating the original problem with the minimization problem of equation (15) the solution yields a solvable, approximated original problem. Thereby $I$ indicates the identity matrix, $\alpha$ the regularization parameter and $x_{0}$ dedicates an a priori estimation of $x$.

$$
\min \left(\|A x-b\|_{2}^{2}+\left\|I \alpha\left(x-x_{0}\right)\right\|_{2}^{2}\right)
$$

With the L-curve the trade-off between the logarithm of the norm of the regularized solution and the corresponding residual norm is rated. In Fig. 2 a typical L-curve is shown. The curve has an L-shape with the optimal $\alpha$ parameter at the bend.

The y-axis shows the variation of the regularized solution. The x-axis, with $x_{\alpha}$ as the approx. solution of the regularization, displays the deviation of $A x_{\alpha}$ to the vector $b$ due to the regularization parameter $\alpha$. The regularization parameter $\alpha$ changes thereby the solution of the original problem. With a high value of $\alpha$, the solution converges to $x_{0}$ and will not fit to the given data. Without a priori knowledge, $x_{0}$ is set to zero. With a small or zero parameter $\alpha$, the fit is close to the original problem, but the contributions from the data errors dominate the solution. 
Considering the different valid switching states of the inverter, the different operations and the regularization the calculation yields reliable parameter results. Not enough valid "active states" and "freewheeling state" makes a manipulation of the pulse pattern as described before necessary, resulting in enough dqequations. Evaluation of these then delivers reliable parameters for each control period. But inducing these PWM excitations causes unsymmetrical switching and probably harmonics. But compared to signal injection within a control period as described in e.g. [4] these harmonics are smaller.

In simulation the parameters are evaluated in each control period $T_{\mathrm{C}}$ with the described algorithm, the simulation results are displayed in the corresponding section.
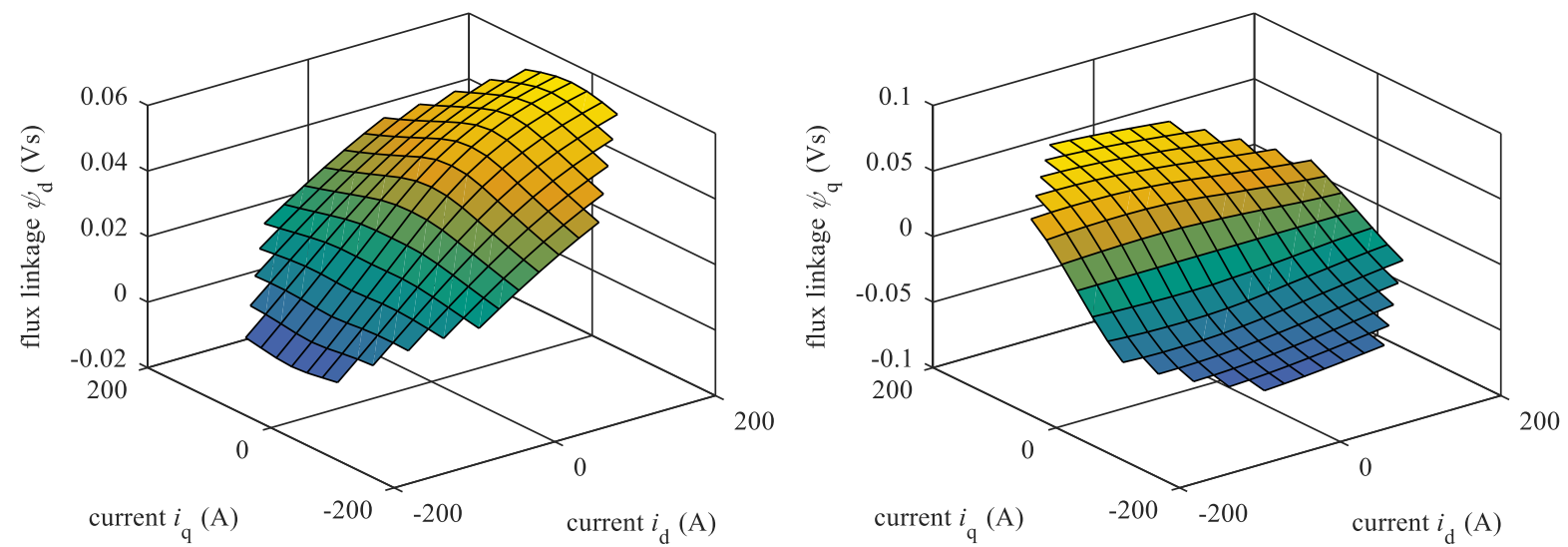

Fig. 3: Direct and quadrature flux linkages of the PSM, calculated by FEA simulation.

\section{Simulation Environment}

In Fig. 4 a typical application or test-bench setup of a PSM machine is shown. The electrical machine is thereby directly connected to a mechanical load. The DC link supplies the two level voltage source inverter. The three phase currents, the DC link voltage and the rotor angle are assumed as directly measured. Due to the challenging measurement of the inverter voltages within a PWM period the inverter voltages are estimated. For the introduced algorithm average voltages within a PWM period are sufficient. These voltages can be determined in e.g. a double

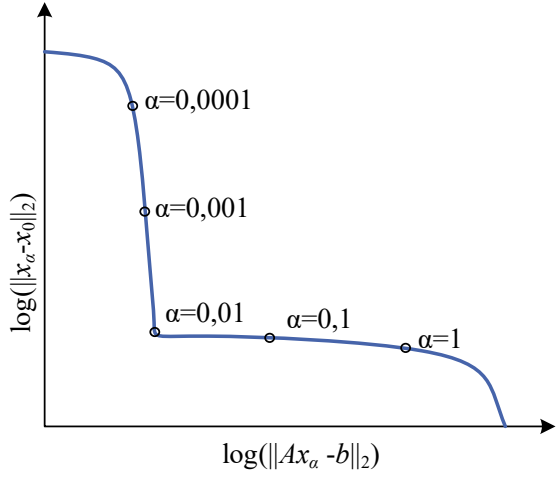

Fig. 2: The L-curve: A logarithmic plot of the norm of a regularized solution vs. the norm of the corresponding residual norm.

pulse measurement [19]. The characteristics for the inverter MOSFET and diode voltage drop at operational conditions, depending on the switching state are stored in lookup tables. All measured and estimated three phase quantities are transformed together with the rotor angle to the rotor-oriented dqframe values. In the simulation environment, the PSM machine is implemented as an acausal Matlab/Simulink-Matlab/Simscape model based on the equations (1) and (2). The model is parametrized with the in Fig. 3 shown finite element analysis (FEA) flux linkages, the stator resistance and the pole pairs. The mechanical load in the simulation is modelled as an ideal speed source. The inverter and signal processing hardware is assumed to be ideal to focus on the identification algorithm. The 
parameters like switching frequency, etc. are thereby derived from the target system. A possible high frequency switching Silicon Carbide inverter with fast current sensors as well as a powerful real-time signal processing hardware are introduced in [20]. This system enables high dynamics and permit the assumption of constant inductances and piecewise linear flux linkages within small control and switching periods. For the current-derivative signal a planar Rogowski coil sensor as shown in [6] is assumed. The different current-derivative sensors are evaluated in [6], the planar Rogowski coil thereby shows high bandwidth and good signal quality for the intended use. In Tab. I the simulation parameters are listed.

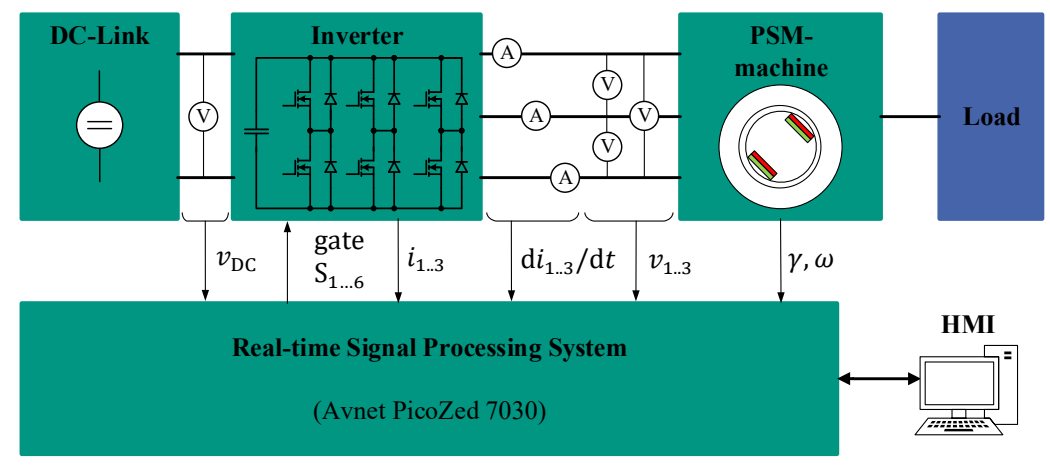

Fig. 4: Typical PSM application with necessary peripheral units and the load.

\section{Simulation Results}

The simulation results in Fig. 5 show the nonlinear parameter calculation during a speed change of 5000 rpm within $0.01 \mathrm{~s}$, thereby the torque is controlled to $20 \mathrm{Nm}$ (approx. $-10 \mathrm{~A} \mathrm{~d}$-axis and $20 \mathrm{~A}$ q-axis current). At $0.13 \mathrm{~s}$ the torque is changed to $-20 \mathrm{Nm}$, at $0.02 \mathrm{~s}$ back to $20 \mathrm{Nm}$. The parameters shown in Fig. 5 show the identified parameters of each control period $T_{\mathrm{C}}$. The difference between the blue calculated parameters and the green dashed model parameters are small at even full dynamic identification. The necessary values of the current derivative, the voltages, the electric angular and the currents are directly evaluated from the simulation. Control periods with prohibited voltage vectors are ignored due to the high dynamics of the algorithm. The parameters show quasi-constant values over the whole operation area. Due to mostly dynamic operation and evaluable dq-equations in this simulation setup, a shift of the pulse pattern is not necessary. The inverse problem is solved at high speed within a simplified matrix as seen at the identification of the resistance at about $0.012 \mathrm{~s}$. The same is done with the flux-linkage identification at times smaller than $0.003 \mathrm{~s}$ with a simplified matrix. In the other cases the inverse problem is solved with the singular value decomposition and an optional regularization for these ill conditioned problems. The results shown in Fig. 5 are without any additional filtering. The parameter peaks in the identification are due to the commutation of the different parts of the identification algorithm depending on the point of operation. For final implementations additional filtering is necessary. Further simulations with equivalent setup have similar results and not displayed to enhance readability. At operation points without enough dq-equations, the evaluation of the parameters is not direct possible. In this case the pulse pattern of the space vector modulation has to be modified as described before, the precise effects of this for the identification approach, will be on further research. The results presented in this paper are sufficient for e.g. adaptive controller lookup tables or condition monitoring without the real-time requirement as a high performance control.

\section{Summary}

This paper shows the derivation of an extended and fast online identification method for the fluxlinkages, the inductances and the stator resistance. The algorithm evaluates the inverter induced current slopes, the currents, the electrical frequency and the applied voltages only with use of the existing PWM excitation. In this paper the different operation points, the unidentifiable voltage vectors and points of operation are described and motivated with the condition number as an indicator for the identifiability. The possible unidentifiable periods are discussed and a solution is presented. The simulation of the 
implemented algorithm, at valid periods, is shown at a certain point of operation. Thereby the simulation environment is parametrized with the test-setup data for future measurements. The simulation results show fast convergence and reliable identification of the parameters at standstill, transient and steadystate operation.
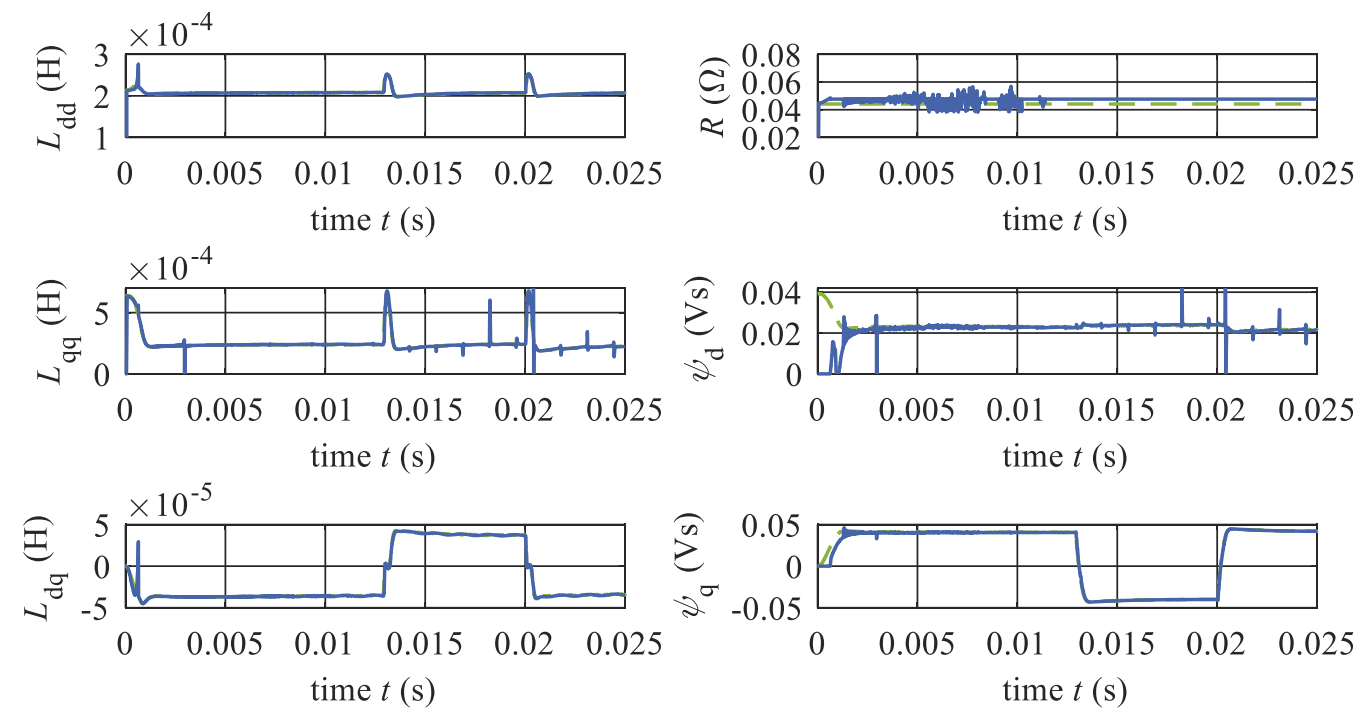

Fig. 5: Stable parameter identification with the explained algorithm for a defined simulation setup. The blue lines are the parameters calculated by the identification algorithm, the green dashed lines are the reference values from a previous FEA simulation (Fig. 3) and mostly covered by the blue identified parameters.

\section{References}

[1] T. Boileau, N. Leboeuf, B. Nahid-Mobarakeh, and F. Meibody-Tabar, "Online Identification of PMSM Parameters: Parameter Identifiability and Estimator Comparative Study," IEEE Trans. on Ind. Applicat., vol. 47, no. 4, pp. 1944-1957, 2011.

[2] J. Noel Hernandez Perez, O. Sandre Hernandez, R. Morales Caporal, J. d. J. Rangel Magdaleno, and H. Peregrina Barreto, "Parameter Identification of a Permanent Magnet Synchronous Machine based on Current Decay Test and Particle Swarm Optimization," IEEE Latin Am. Trans., vol. 11, no. 5, pp. 1176-1181, 2013.

[3] S. A. Odhano et al., "Parameter Identification and Self-Commissioning in AC Motor Drives: A Technology Status Review," IEEE Trans. Power Electron., p. 1, 2018.

[4] J. Bonifacio, N. Amann, and R. Kennel, "Online full-parameter identification strategy for IPMSM using voltage signal injection," in Proceeding of the 20th European Conference on Power Electronics and Applications - EPE 2018 ECCE Europe, 2018.

[5] S. Bolognani, S. Calligaro, R. Petrella, and M. Sterpellone, "Sensorless control for IPMSM using PWM excitation: Analytical developments and implementation issues," in 2011 Symposium on Sensorless Control for Electrical Drives, Birmingham, United Kingdom, 2011, pp. 64-73.

[6] S. Decker, A. Liske, Schweiker, Kolb, Johannes, Daniel, and M. Braun, "Measurement of Two-Level Inverter Induced Current Slopes at High Switching Frequencies for Control and Identification Algorithms of Electrical Machines," in 2018 International Power Electronics Conference (IPEC-Niigata 2018 - ECCE ASIA), Niigata, 2018, pp. 1-6.

[7] M. X. Bui, M. F. Rahman, and D. Xiao, "Sensorless Position Estimation, Parameter Identification and Control Integration for Permanent Magnet Synchronous Machines using Current Derivative Measurements," 
in 2018 International Power Electronics Conference (IPEC-Niigata 2018 - ECCE ASIA), Niigata, 2018, pp. 1-7.

[8] H. Li, J. Melkebeek, and F. de Belie, "Current Response Based Online Inductance Estimation Method for IPMSM DTC Drives Considering Magnetic Saturation," in 2018 International Symposium on Power Electronics, Electrical Drives, Automation and Motion (SPEEDAM), Amalfi, Italy, 2018, pp. 39-44.

[9] B. Stumberger, G. Stumberger, D. Dolina, A. Hamler, and M. Trlep, "Evaluation of saturation and crossmagnetization effects in interior permanent magnet synchronous motor," in Conference Record of the 2001 IEEE Industry Applications Conference. 36th IAS Annual Meeting (Cat. No.01CH37248), Chicago, IL, USA, 2001, pp. 2557-2562.

[10] J. A. Melkebeek and J. L. Willems, "Reciprocity relations for the mutual inductances between orthogonal axis windings in saturated salient-pole machines," IEEE Trans. on Ind. Applicat., vol. 26, no. 1, pp. 107$114,1990$.

[11] Le Chang and T. M. Jahns, "Prediction and Evaluation of PWM-Induced Current Ripple in IPM Machines Incorporating Slotting, Saturation, and Cross-Coupling Effects," IEEE Trans. on Ind. Applicat., vol. 54, no. 6, pp. 6015-6026, 2018.

[12] S. A. Odhano, R. Bojoi, S. G. Rosu, and A. Tenconi, "Identification of the magnetic model of permanent magnet synchronous machines using DC-biased low frequency AC signal injection," in 2014 IEEE Energy Conversion Congress and Exposition (ECCE), Pittsburgh, PA, USA, 2014, pp. 1722-1728.

[13] J. Richter, T. Gemassmer, and M. Doppelbauer, "Predictive current control of saturated cross-coupled permanent magnet synchronous machines," in 2014 International Symposium on Power Electronics, Electrical Drives, Automation and Motion, Ischia, Italy, 2014, pp. 830-835.

[14] S. Decker, J. Richter, and M. Braun, "Predictive current control and online parameter identification of interior permanent magnet synchronous machines," in 2016 18th European Conference on Power Electronics and Applications (EPE'16 ECCE Europe), Karlsruhe, 2016, pp. 1-10.

[15] Q. Gao, G. M. Asher, M. Sumner, and P. Makys, "Position Estimation of AC Machines Over a Wide Frequency Range Based on Space Vector PWM Excitation,” IEEE Trans. on Ind. Applicat., vol. 43, no. 4, pp. 1001-1011, 2007.

[16] A. Tarantola, Inverse problem theory and methods for model parameter estimation. Philadelphia, Pa.: SIAM - Soc. for Industrial and Applied Math, 2005.

[17] P. C. Hansen, Rank-Deficient and Discrete Ill-Posed Problems: Society for Industrial and Applied Mathematics, 1998.

[18] P. C. Hansen, "The L-Curve and its Use in the Numerical Treatment of Inverse Problems," in in Computational Inverse Problems in Electrocardiology, ed. P. Johnston, Advances in Computational Bioengineering, 2000, pp. 119-142.

[19] F. Stamer, R. Schwendemann, and M. Hiller, “A multi-dimensional full automatic power semiconductor test bench for accurate semiconductor loss calculation," in International Exhibition and Conference for Power Electronics, Intelligent Motion, Renewable Energy and Energy Management Proceedings (PCIM Europe 2019), 2019, pp. 1411-1418.

[20] R. Schwendemann, S. Decker, M. Hiller, and M. Braun, “A Modular Converter- and Signal-ProcessingPlatform for Academic Research in the Field of Power Electronics," in 2018 International Power Electronics Conference (IPEC-Niigata 2018 - ECCE ASIA), Niigata, 2018, pp. 1-7. 